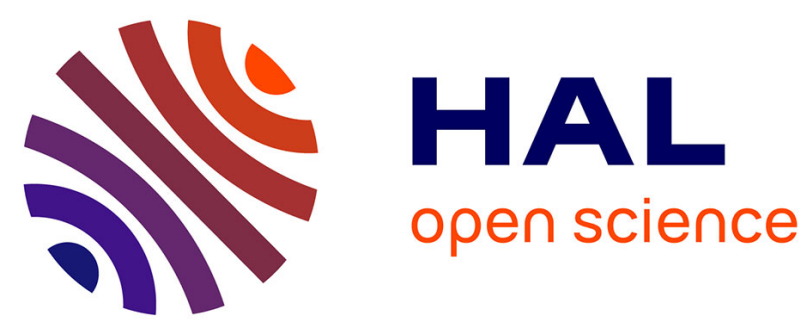

\title{
Open Access Digital Tools' Application Potential in Technological Process Planning: SMMEs Perspective
}

Roman Wdowik, Chandima R.M. Ratnayake

\section{To cite this version:}

Roman Wdowik, Chandima R.M. Ratnayake. Open Access Digital Tools' Application Potential in Technological Process Planning: SMMEs Perspective. IFIP International Conference on Advances in Production Management Systems (APMS), Sep 2019, Austin, TX, United States. pp.312-319, 10.1007/978-3-030-29996-5_36. hal-02460471

\section{HAL Id: hal-02460471 \\ https://hal.inria.fr/hal-02460471}

Submitted on 30 Jan 2020

HAL is a multi-disciplinary open access archive for the deposit and dissemination of scientific research documents, whether they are published or not. The documents may come from teaching and research institutions in France or abroad, or from public or private research centers.
L'archive ouverte pluridisciplinaire HAL, est destinée au dépôt et à la diffusion de documents scientifiques de niveau recherche, publiés ou non, émanant des établissements d'enseignement et de recherche français ou étrangers, des laboratoires publics ou privés.

\section{(c)(1)}

Distributed under a Creative Commons Attribution| 4.0 International License 


\title{
Open Access Digital Tools' Application Potential in Technological Process Planning: SMMEs Perspective
}

\author{
Roman Wdowik $^{1}$ and R.M. Chandima Ratnayake ${ }^{2}$ \\ ${ }^{1}$ Rzeszów University of Technology; The Faculty of Mechanical Engineering and Aeronautics, \\ 35-959 Rzeszów, Poland \\ rwdowik@prz.edu.pl \\ ${ }^{2}$ University of Stavanger, Department of Mechanical and Structural Engineering and Materials \\ Science, N-4036 Stavanger, Norway \\ chandima.ratnayake@uis.no
}

\begin{abstract}
This concept study focuses on technological process planning (TPP), taking into account the application potential of open access digital tools (OADT) in small- and medium-scale manufacturing enterprises (SMMEs). It presents the authors' classification of digital tools (DT) used in the SMMEs and available groups of OADT. It also proposes possible scenarios' potential for future TPP by taking into account the developments in artificial intelligence (AI) and immersive technologies, i.e. virtual and augmented realities (VR/AR). It also focuses on challenges and procedures regarding the implementation of DT in specific SMMEs' environments, focusing on how open access tools play a crucial role at the first stages of SMME development, as these tools enable minimization of resource wastage. Although the capabilities of these tools are limited, it is vital to develop implementation strategies within a SMME, based on specific need(s).
\end{abstract}

Keywords: Digital Tools, Open Access, Small-Medium Scale Manufacturing Enterprises, Process Planning, Digitalization.

\section{Introduction}

\subsection{Motivation}

Digital transformation, such as: cloud data computing techniques (CDCT), machine learning (ML), virtual reality (VR), cloud-based tools (CBT), augmented reality (AR), artificial intelligence (AI), data-driven production control, etc., offer new opportunities to enhance the operational performance of mechanical and manufacturing engineering $[1,4,9]$. In addition, fast-growing digital transformation leads to a demand for more personalized, connected, smart, and sustainable products and services [2]. Although the availability of information is immense, due to digitalization, it is very important to share and use it through the effective use of computer networks and at the lowest possible cost (e.g. by the use of open access tools) [3]. Although the aforementioned is novel, there is significant level of challenges which exist in small- and medium-scale manufacturing enterprises (SMMEs), especially in the implementation into their shop floors 
of the newest, fast-growing digital technology, often with limited funds and availability of other infrastructure resources such as human resources, R\&D and training centers, etc.

Technological process planning (TPP) plays a vital role in manufacturing environments, due to the fact that it can be considered one of the most important stages of production, especially from an overall productivity enhancement perspective [5]. TPP provides a foundation for all manufacturing success, leading to the definition of machining operations' order, choice of necessary fixtures, tools and parameters of processes which are run using CNC machine tools and technological equipment [6]. Although the efforts regarding the implementation of CAPP tools [7] and CAD/CAMs [8] are well known, and these digital tools have proved their usability in digital transformation, contemporary advanced digital and smart manufacturing redefines overall success in relation to the optimization of: design, production, supply chain and customer service perspectives [7]. In this context, it is vital to investigate how to reinvent TPP to cater for the latest and expected future needs and how to enable the expansion of startups and SMMEs.

To reinvent TPP to be efficient and effective requires making use of expert knowledge, together with artificial intelligence (AI), databases, together with machine learning techniques, as well as analysis of market trends and the implementation of various digital tools (DT) such as software and/or devices. In addition, cloud-based techniques could be used [9]. In this context, SMMEs need appropriate strategies and plans, in order to accelerate their development and to be able to compete with potential competitors. Various aspects enable the aforementioned to be achieved. For instance, currently there is a trend to use digital tools with common software platforms (e.g. 3D systems, Autodesk ${ }^{\circledR}$ BIM 360 Design, etc.) to share the data, accelerate TPP, test the prototypes (i.e. in a physical or virtual environment) or operate existing hardware (e.g. CNC machine tools). In this context, SMMEs must make a decision regarding investments and, at the early stage of their operation, whether they need to use open access (free or low-cost) digital tools or to buy other tools existing in the market. Hence, this manuscript mainly presents findings and suggestions, focusing on the use of digital tools in SMMEs, and provides a concise discussion on existing possibilities for the use of digital tools in SMMEs, which use several CNC machine tools in a manufacturing environment. Furthermore, it takes into account the groups of digital tools, their examples, limitations and approaches for their use in TPP. In addition, it suggests the potential to increase R\&D work, focusing on free digital tools.

\subsection{Classification of Digital Tools used in TPP}

The digital tools, which are used in TPP, may be classified into groups such as: Software tools (S), e.g. computer applications; Devices (D), e.g. PCs, smartphones, tablets, smartwatches, etc.; machines, machine tools and technological equipment (MTE), e.g. CNC machine tools; technological equipment (e.g. tools, chucks, presetters, etc.); and Internet tools (IT), e.g. webpages, webguides, webcatalogues, internet calculators, internet clouds, internet search engines, etc. The classification proposed by the authors is presented in Fig. 1. It classifies the most important DT on the basis of expert 
knowledge, experience and discussion with industrial partners. However, the number of DT in SMMEs is limited compared to those in large firms, due to the costs generated by DT.

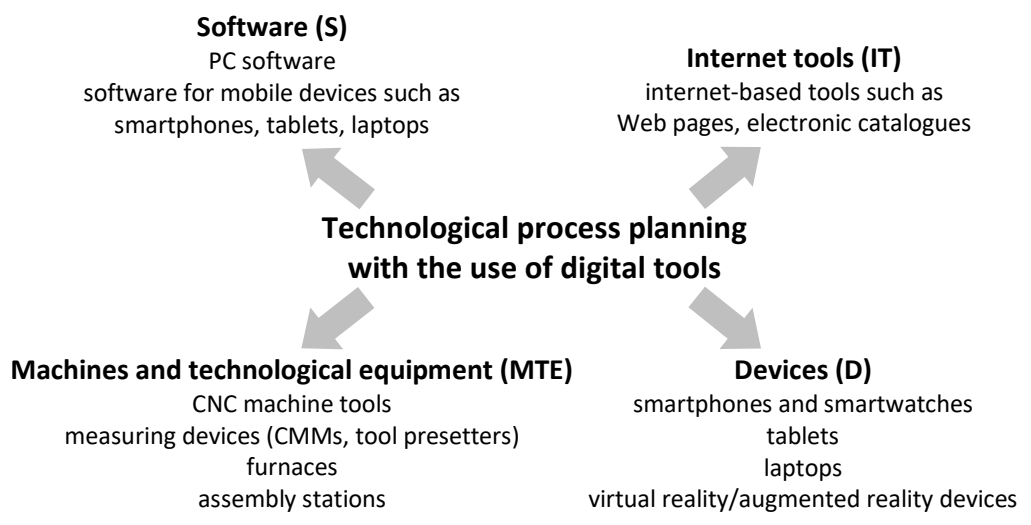

Fig. 1. Classification of the groups of digital tools used in TPP.

\subsection{Perspectives of Technological Process Planning in SMMEs}

There are several approaches in TPP which can be considered by industrial companies (refer to Fig. 2). The first one, standard process planning, mainly uses the expert knowledge of a process planner and is a combination of experience, tests and the use of guidelines. It has been used for many decades by process planners. SMMEs which, in general, are careful when they face new investments, have so far used this approach. In the authors' opinion, it is mainly caused by availability and low expenditure. Recent decades have proved that the application of CAD/CAM systems may help to eliminate paper documentation, accelerate and automate the manufacturing processes of complex parts and provide great opportunity for safer (digital) simulation of manufacturing operations which will be run on real machines.

In parallel with the abovementioned two approaches, it can be beneficial to invest in completely new solutions for TPP, which are based on immersive technology (e.g. VR) and AI. Immersive technology may provide the opportunity to prepare and test technological processes in a virtual environment, while AI may lead to automation. It can be adapted both to VR/AR and standard approaches. Fig. 2 presents the level of complexity of the aforementioned approaches and shows approximate, time-related implementation and possible future scenarios.

In the case of SMMEs, the use of immersive technology may be beneficial because it still provides an opportunity to use expert knowledge. However, all TPP tasks are to be performed in one virtual environment. The limited resources of SMMEs are, in this case, easier to implement into VR than in the case of large enterprises. In addition, in the case of various SMMEs, immersive testing of manufacturing processes seems to be more important than automation - mainly if open access virtual realities were to be 
delivered. AI may play a crucial role in less complex manufacturing tasks and infrastructures (e.g. SMMEs' infrastructures), which do not require advanced expert knowledge and the analysis of many variables and external noise, and also the implementation of AI algorithms should be cost-effective.

The next years will also be important because of the implementation of faster communication techniques. The new trend, which can now be observed regarding the implementation of $5 \mathrm{G}$ mobile networks may be very beneficial for SMMEs. This new technology may allow the company to be managed more efficiently. The $5 \mathrm{G}$ networks could provide the opportunity, e.g. in several years/decades, to start many SMMEs. The infrastructures of SMMEs will be susceptible to wireless communication. In this context, resources existing in different places will be connected and used for the aims of new SMMEs' development. This will be very important at the start of an SMME. Connecting advanced manufacturing resources is much faster in this case.

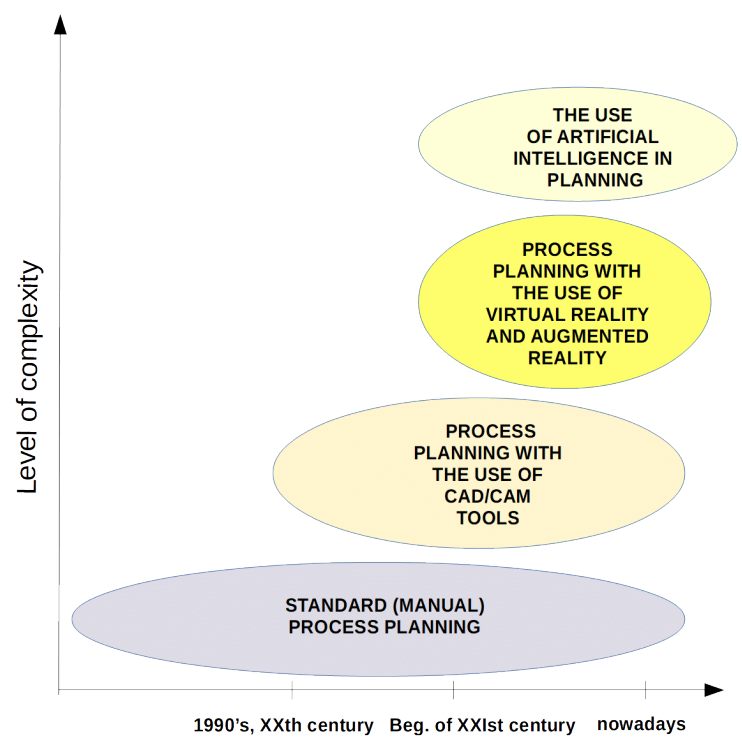

Fig. 2. The development of TPP applications and possible future scenario.

\section{Existing Open Access Digital Tools and their Availability for SMMES}

The main issue regarding open access digital tools (OADT) concerns the effective strategies of their use in SMMEs. Table 1 presents the main groups of open access tools for TPP. Their main functionalities and examples are also presented. In the authors' opinion, the OADT are limited to Software (S) and Internet tools (IT). However, SMMEs must invest funds in Devices (D) and Machine tools/technological equipment (MTE). It can be observed that different CAD digital tools, CAM digital tools and digital calculators (i.e. enabling machining parameters' selection and verification) are available 
in open access mode both as stand-alone licenses on PCs, smartphones, tablets, and as Internet tools. Usually their functionalities are limited, compared to commercial software (i.e. CNC programming is limited to 2 or 2.5 axes, 3D modelling is less effective or impossible, etc.), but they can be considered the first choice if the existing budget is used for the purchase of machine tools and technological equipment. In the case of commercial software used by large/developed companies, it is reasonable to use the software offered by one producer, taking into account the fast training of employees. However, the bankruptcy of a producer will cause additional expenses for a large company. If SMMEs want to use open access tools, they must arrange a list of digital tools, which consist of the products of different companies. Developing the proper training procedures will protect an SMME against unwanted expenditure.

As the conclusion of this chapter it should be stated that OADT find wider application in SMMEs than in large enterprises, but it could also be changed by increased (several times) R\&D and dissemination of digital techniques.

Table 1. The main groups and examples of OADT for TPP in SMMEs.

\begin{tabular}{lll}
\hline & Main functionalities & Examples \\
\hline & $\begin{array}{l}\text { 2D drawing preparation, viewing of 2D or } \\
\text { 3D models, measurements of dimensions, } \\
\text { printing, changing a file format, copying or } \\
\text { pasting CAD data, error correction, etc. }\end{array}$ & $\begin{array}{l}\text { For PC: DraftSight, } \\
\text { FreeCAD, Libre CAD } \\
\text { For Mobile Devices: } \\
\text { CAD Reader, CAD Assis- } \\
\text { tant }\end{array}$ \\
\hline & $\begin{array}{l}\text { Basic 2-, 2.5- or 3-axis machining, simula- } \\
\text { tion of tool paths, basic postprocessing capa- }\end{array}$ & $\begin{array}{l}\text { For PC: CamBam (par- } \\
\text { tially free), FreeMILL, G- } \\
\text { bilities, etc. }\end{array}$ \\
\hline & $\begin{array}{l}\text { Calculations of power requirement in ma- } \\
\text { chining, the choice of depths of cut, rough- } \\
\text { ness, dressing conditions for grinding, etc. }\end{array}$ & $\begin{array}{l}\text { Web/Mobile Apps: Walter } \\
\text { feed and speeds, Dr Kai- } \\
\text { ser App }\end{array}$ \\
\hline & $\begin{array}{l}\text { Choice of tools and machining parameters, } \\
\text { data sharing by the use of network, search- } \\
\text { ing data, etc. }\end{array}$ & $\begin{array}{l}\text { Tool Guide, Iscar Tool } \\
\text { Advisor }\end{array}$ \\
\hline
\end{tabular}

\section{The Proposed OADT Implementation Procedures}

Implementation of OADT requires the proper procedures (see Fig. 3). Approach I is the standard procedure, which is proposed at an early stage of an SMME's existence. If OADT cannot be implemented in the specific manufacturing environment of the SMME, traditional TPP (without the use of digital solutions) may be developed. While the SMME grows and money savings exist (e.g. they can be used for new investments), additional possibilities should be considered (Approach II). However, these possibili- 
ties require detailed risk calculations and cost-effective investments in short-term software rent, license purchase or subcontracting of the SMME's contracts by other companies. The further development of the SMME may lead to the final purchase of commercial DTs. However, the development of used OADT at an early stage may also be sufficient to meet all the main requirements of the SMME without investing in commercial software in the future. This concept generates added value by promoting concentration on innovations in the area of OADT development in the SMME (e.g. investments in new functions of existing OADT or new open access tools).

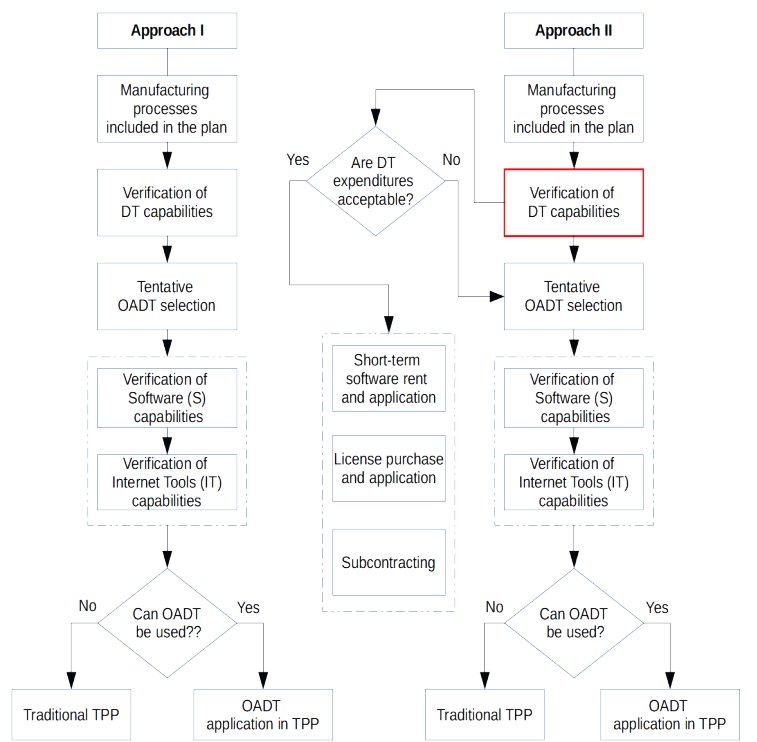

Fig. 3. The approaches of OADT application in TPP of SMMEs.

\section{Cost-related Implementation of the Digital Tools in SMMEs}

The cost of digital tools $\left(\mathrm{C}_{\mathrm{DT}}\right)$ is the sum of machine and technological equipment costs $\left(\mathrm{C}_{\mathrm{MTE}}\right)$, software costs $\left(\mathrm{C}_{\mathrm{S}}\right)$, Internet tools' costs $\left(\mathrm{C}_{\mathrm{IT}}\right)$ and devices' costs $\left(\mathrm{C}_{\mathrm{D}}\right)$ :

$C_{D T}=C_{M T E}+C_{S}+C_{I T}+C_{D}$

Usually, in the case of manufacturing firms, the number of DT increases as the firm grows. Fig. 4 presents this in the case of a small enterprise (SE), a medium enterprise (ME) and a large enterprise (LE). It is caused by the increased expectations from customers and also internal developments. The increase in the number of DT may cause an increase in the use of OADT. Which tools their employees should use is usually the decision of managers, but very often nowadays employees may decide how to enhance their work efforts by the use of new open access applications. It is a win-win situation in the case of SMMEs, which do not define rigid procedures, for both employees and employers. The employer does not need to invest additional funds, and the employee 
can contribute to the firm's growth by using OADT. Because of the increased number of DT in the case of a larger company, the expenses (DT expenses) may also increase. The expenses should be balanced by the proper income, in order to obtain adequate profit. In the case of SMMEs, the cost of preparation of a technological process can be defined as high. In this context, the technological process can be understood as a product, which costs less if mass-produced.

On the basis of the abovementioned discussion, it can be stated that, in the case of SMMEs, a reduction in expenses regarding technological process planning may be realized by the use of open access digital tools, even if the total number of existing digital tools is limited. Moreover, added value may be generated if the development process is also focused on OADT capabilities.
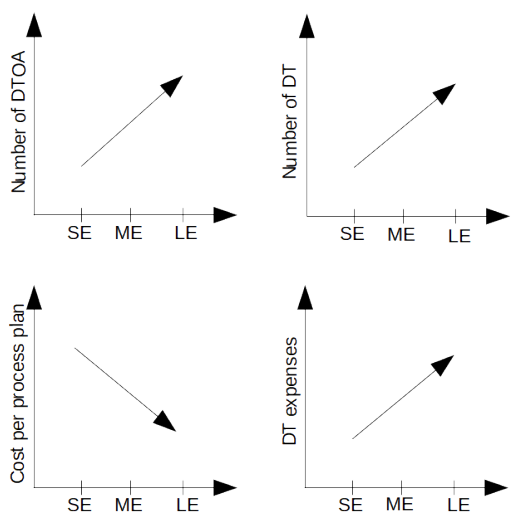

Fig. 4. Comparison of selected and expected DT-related indicators in small (SE), medium (ME) and large (LE) enterprises.

Fig. 5 presents the challenges (aims) of SMMEs regarding the implementation of digital tools. It can be stated that the main challenges concern reducing expenses, automation, data sharing, training designed for employees, distance work, new investments and investors, co-funding capabilities and promotion. Next to the abovementioned aims, Fig. 5 presents the groups of digital tools which can be useful in achieving the specified aims. The groups of products which can be offered by SMMEs are also presented. In this context, manufacturing firms can offer digital data, sub-contracting, service and other products.
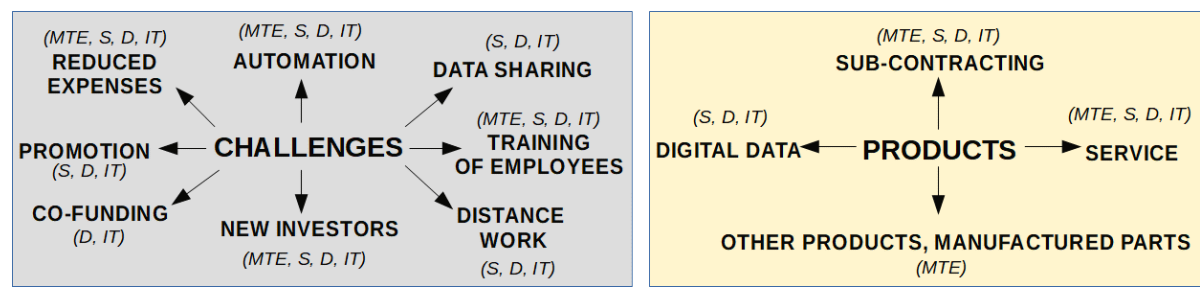

Fig. 5. Challenges of CNC machine tools-based SMMEs and products offered with groups of digital tools used for specific aims (refer to Fig. 1). 


\section{Conclusion}

SMMEs usually need additional resources for investments. The use of DT, available as commercial licenses, is possible while SMMEs develop and gather the funds. At the early stage of SMMEs' existence, they need the support from open access digital tools (OADT). OADT have functional limitations but are offered for free or at a relatively low price and can be researched for the specific needs of the SMME. This study is a concise analysis of OADT. The OADT could be applied by SMMEs using the proposed implementation procedures, also by taking into account the presented capabilities.

\section{Acknowledgement}

This study was developed within the project, "Science internship for the investigations of digitalization in manufacturing", financed by the Polish National Agency for Academic Exchange (www.nawa.gov.pl) in the Bekker Programme.

\section{References}

1. Chryssolouris, G., Mavrikios, D., Papakostas, N., Mourtzis, D., Michalos, G., Georgoulias, K.: Digital manufacturing: history, perspectives, and outlook. Proceedings of the Institution of Mechanical Engineers Part B Journal of Engineering Manufacture, 2009, https://doi.org/10.1243/09544054JEM1241.

2. Moghaddam, M., Cadavid, M. N., Kenley, C.R., Deshmukh, A.V.: Reference architectures for smart manufacturing: A critical review. Journal of Manufacturing Systems (49), 215-225 (2018), https://doi.org/10.1016/j.jmsy.2018.10.006.

3. Calvo, I., López, F., Zulueta, E., González-Nalda, P.: Towards a methodology to build virtual reality manufacturing systems based on free open software technologies. International Journal on Interactive Design and Manufacturing 11, 569-580 (2018), http://doi.org/10.1007/s12008016-0311-x.

4. Enzo, M., Frazzon, E.M., Kück, M., Freitag, M.: Data-driven production control for complex and dynamic manufacturing systems. CIRP Annals 1(67), 515-518 (2018), https://doi.org/10.1016/j.cirp.2018.04.033.

5. Wdowik, R., Magdziak, M., Ratnayake, R.M.C., Borsellino, Ch.: Application of process parameters in planning and technological documentation: CNC machine tools and CMMs programming perspective. Procedia CIRP (78), 43-48 (2018), https://doi.org/ 10.1016/j.procir.2018.09.054.

6. Feld, M.: Basics of technological process planning of machines' parts (in Polish). Wydawnictwa Naukowo-Techniczne, Warsaw (2003).

7. Zhang, H.-C.: Computer Aided Process Planning: The state-of-the-art survey. International Journal of Production Research 4(27), 553-585 (1989), http://doi.org/10.1080/00207548908942569.

8. Kutin, A., Dolgov, V., Sedykh, M., Ivashin, S.: Integration of different computer-aided systems in product designing and process planning. Procedia CIRP (67), 476-481 (2018), https://doi.org/10.1016/j.procir.2017.12.247.

9. Tarchinskaya, E., Taratoukhine, V., Matzner, M.: Cloud-based engineering design and manufacturing: State-of-the-art. 7th IFAC Conference on Manufacturing Modelling, Management, and Control International Federation of Automatic Control, June 19-21, Saint Petersburg, Russia (2013). 